\title{
INTRODUCTION \\ Surgery involving the venous system and complication avoidance
}

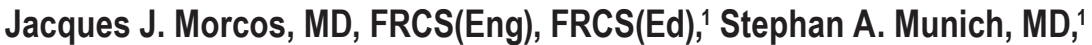 \\ Tiit Mathiesen, MD, PhD, ${ }^{2}$ Marc P. Sindou, MD, PhD, ${ }^{3}$ and Vinko V. Dolenc, MD, PhD ${ }^{4}$
}

${ }^{1}$ Department of Neurological Surgery, University of Miami, Florida; ${ }^{2}$ Department of Neurosurgery, Karolinska Hospital, Stockholm, Sweden; ${ }^{3}$ Department of Neurosurgery, University of Lyon, France; and ${ }^{4}$ Department of Neurosurgery, University Medical Center Ljubljana, Slovenia

$\mathrm{W}$ HILE arterial complications in neurosurgery more often attract the spotlight, venous complications frequently go unrecognized or unappreciated. Until recently, the importance of veins and the avoidance of their related complications simply were overlooked. Aneurysms and vascular malformations occupy the daily consciousness of the cerebrovascular surgeon, yet a thorough appreciation of veins, in health and disease states, is essential not only for cerebrovascular surgeons but equally, if not more so, for tumor surgeons, skull base surgeons, neurotrauma surgeons, interventionalists, and others. With improved microsurgical instruments and techniques and more sophisticated means of assessing venous anatomy, a greater understanding of the significance of cerebral veins is developing. Adding to this understanding is the present issue of Neurosurgical Focus, which includes 11 articles discussing surgeries that involve the venous system and the avoidance of venous complications.

Toda et al. describe a series of 121 patients with trigeminal neuralgia and 205 patients with hemifacial spasm, finding that venous compression portended a worse prognosis than arterial compression following microvascular decompression. The consequence of sacrificing the superior petrosal vein remains an area of controversy. Narayan and colleagues, from Dr. Nanda's group, reviewed 35 publications and found many studies that reported no adverse effects due to superior petrosal vein sacrifice, while others had reported complication rates of up to $30 \%$. Sacrifice of the superior petrosal vein is an example of an intentional venous insult. Yet venous injury may be unintentional, such as in the case of venous sinus thrombosis following surgery. Abou-Al-Shaar et al. found that the incidence of this complication was $6 \%$ after surgery for vestibular schwannomas, and they provide a potential treatment algorithm for its management.

Appreciation of the variability of cerebral venous anatomy is critical to avoidance of venous complications. A 6-point grading scale (1 through 5a and 5b) for the position of the jugular bulb is provided by Dr. Manjila's group. Intraoperative assessment of venous anatomy and flow may also help avoid venous complications. This issue contains two articles describing different techniques for intraoperative assessment: Prada et al. report their use of intraoperative ultrasound, while Acerbi and associates report their use of ICG videoangiography and Flow 800 analysis.

Dr. Manjila and colleagues also provide a nice review of more rare, extraaxial venous anomalies, including persistent embryonic sinuses, sinus pericranii, venous varices, and enlarged emissary veins. The influence of venous sinus stenosis on the pathophysiology of idiopathic intracranial hypertension is presented by Giridharan et al. Cappuzzo et al. report on a series of venous sinus stenting for this pathology, finding that $88.9 \%$ of patients experienced improvement of their preoperative symptoms. Roethlisberger et al. review the risk factors and clinical presentations of cerebral venous thrombosis in women taking oral contraceptives. They found coma at admission, thrombosis of the deep venous system, intraventricular hemorrhage, and hemorrhagic venous infarctions to be associated with increased necessity for surgical intervention.

While much of this issue of Neurosurgical Focus considers the cerebral veins as the surgeon's foes, Chen and associates show us that they can, indeed, also be our friends. These authors provide a thorough review of the technical considerations, indications, and outcomes for transvenous embolization of arteriovenous malformations. 
We hope that this issue of Neurosurgical Focus will add to the growing body of literature highlighting the importance of the cerebral veins, not only as critical structures whose injury is to be avoided (e.g., in venous infarction), but also as avenues of potential therapeutic intervention (e.g., transvenous embolization).

https://thejns.org/doi/abs/10.3171/2018.5.FOCUS18229

\section{Disclosures}

The authors report no conflict of interest. 